\title{
COMPACTNESS OF MAPPINGS ON PRODUCTS OF LOCALLY CONNECTED GENERALIZED CONTINUA
}

\author{
R. F. DICKMAN, JR.
}

Definition. A connected topological space has the complementation property provided the complement of any compact set has at most one nonconditionally compact component.

Throughout this paper let $X$ and $Y$ denote noncompact locally connected generalized continua and let $R$ denote a regular curve, i.e. let $R$ denote a locally connected generalized continuum that has the property that for any point $p$ in $R$ and any open set $U$ of $R$ containing $p$ there exists an open set $V$ of $R$ containing $p$ such that $V \subset U$ and Fr $V$ is a finite set. By a mapping we will always mean a continuous function.

Theorem. The product space $Z=X \times Y$ has the complementation property.

Proof. Let $K$ be a compact set in $Z$ and let $U$ and $V$ be conditionally compact open subsets of $X$ and $Y$ respectively such that $K \subset(U \times V)$. Let $P$ be any nonempty component of $X-\bar{U}$ and let $Q$ be any nonempty component of $X-\bar{V}$. Then the connected set $(P \times Y)+(X \times Q)$ intersects every component of $H=Z-(\bar{U} \times \bar{V})$ and hence $H$ is connected. This implies that $Z-K$ has exactly one nonconditionally compact component.

Corollary 1. Let $f$ be a mapping of $Z=X \times Y$ onto a Hausdorff space $W$ and suppose that point inverses of $f$ have compact boundaries. Then if some point inverse $A=f^{-1}(p)$ is not compact, $H=Z$-int $A$ is a compact set that maps onto $W$ and hence $f$ is a closed mapping.

Proof. Since $F=\operatorname{Fr} A$ is compact and $Z$ is locally connected, there is a nonconditionally compact component $Q$ of $Z-F$ that lies entirely in int $A$. By the theorem above $Q$ is the only nonconditionally compact component of $Z-F$ and thus $Z-Q$ is a compact set. Since $H$ is a closed subset of $Z-Q, H$ is compact.

Corollary 2. Let $f$ be a closed mapping of $Z=X \times Y$ onto a noncompact metric space $W$. Then $f$ is a compact mapping.

Proof. By a well-known result of Vainnšteln, point inverses of $f$ have compact boundaries; thus by Corollary (1), point inverses of $f$

Received by the editors September 29, 1966. 
are compact. Closed mappings with compact point inverses are known to be compact mappings.

Corollary 3. Let $f$ be a mapping of $Z=X \times Y$ into the regular curve $R$ and suppose that point inverses of $f$ have compact boundaries. Then (1) if the closure of $f(Z)$ in $R$ is not compact, $f$ is a compact mapping; and (2) if the closure of $f(Z)$ in $R$ is compact, then for any compactification $C$ of $Z$ there is a continuous extension of $f$ to all of $C$.

PROOF OF (1). Suppose that $f$ is not compact. Then there exists a sequence $\left\{x_{i}\right\}$ in $Z$ such that $\left\{x_{i}\right\}$ does not have any convergent subsequences and such that $\left\{f\left(x_{i}\right)\right\}$ converges to some point $y$ in $R$. Let $V$ be any conditionally compact open set containing $y$ such that $F=\mathrm{Fr} V$ is a finite set. By Corollary (1) $K=f^{-1}(F)$ is a compact set. Furthermore since $P=f^{-1}(V)$ is not conditionally compact, $P$ contains a nonconditionally compact component of $Z-K$ and since the closure of $f(Z)$ is not compact, $Q=f^{-1}(R-\bar{V})$ is not conditionally compact and hence also contains a nonconditionally compact component of $Z-K$. But this is a contradiction since $Z$ has the complementation property. Hence $f$ is a compact mapping.

Part (2) is a consequence of Theorem (3.1) of [1].

COROLlary 3.1. Let $f$ be a real-valued mapping defined on $X \times Y$ such that boundaries of point inverses are compact. Then (1) if $f$ is not bounded, $f$ is a compact mapping; and (2) if $f$ is bounded, then for any compactification $C$ of $X \times Y$ there is a continuous extension of $f$ to all of $C$.

Corollary 4. Let $f$ be any reflexive compact mapping of $Z=X \times Y$ onto $E^{2}$, i.e. let $f$ be any mapping of $Z$ onto $E^{2}$ such that for any compact set $A$ in $Z, f^{-1} f(A)$ is also compact. Then $f$ is a compact mapping.

Proof. The corollary is a consequence of the above theorem and Theorem 6 of [2].

Corollary 4.1. Let $f$ be any 1-1 mapping of $Z=X \times Y$ onto $E^{2}$. Then $f$ is a homeomorphism.

\section{REFERENCES}

1. R. F. Dickman, Jr., Unicoherence and related properties, Duke Math. J. 31 (1967), 343-352.

2. E. Duda, Reflexive compact mappings, Proc. Amer. Math. Soc. 17 (1966), 688-693.

UNIVERSITY OF MiAMI 